\title{
On meetings that matter
}

\section{David Albertini}

Published online: 13 May 2010

(C) Springer Science+Business Media, LLC 2010

For clinicians and scientists, attending meetings plays a central role in career development and, in turn, influences the role these health care professionals will ultimately play in the advancement of medical care. Meetings become a way of life in the biomedical research enterprise and have undergone a noticeable evolution: from what were once didactic scholarly events often held in urban landscapes, to the mass gatherings that now fill spacious conference halls in settings chosen for their tourist appeal.

I recall (as a graduate student) attending my first major conference in San Juan, Puerto Rico: I was greeted on the tarmac with Pina Coladas, and thrilled to be escaping the late-November onset of winter in Boston! Even reflecting, some 30 years later, upon the many venues and experiences I've racked up over the years has not deterred in the least bit from that primal experience, from which I first acquired an appetite for the combination of science and sunshine. Not surprisingly, there was a moratorium placed on holding this particular meeting in warm and sunny venues for the next 20 years, due in large measure to the poor attendance at many of the meeting sessions. But in general, we take to these destinations looking not for a change in climate, but for an intense round of networking with comrades and colleagues alike, a chance to accumulate new knowledge by rerouting our brain circuitry in the specialized areas in which we work.

Medical science does not stand still. The pace and intensity of discovery and translation has only increased over the last 30 years, and as scholars and practitioners, meetings are the major forum at which we expand our knowledge base.

D. Albertini $(\bowtie)$

University of Kansas Medical Center,

Kansas City, KS 66103, USA

e-mail: dalbertini@kumc.edu
As most of you well know, every once in a while you'll attend a meeting and realize that it has "breakthrough" written all over it. Setting the stage for such an event is a rare confluence of factors: The alignment of a unique set of individuals (typically experts with diverse backgrounds); a truly multidisciplinary environment of open discourse; and a dire need for solutions to major scientific problems. Such was the case for the first World Congress on Fertility Preservation held in Brussels in December of 2009.

After nearly 30 years of advances in reproductive medicine and the maturation of "bench-to-bedside" practice for the treatment of disorders in human reproduction, the field of fertility preservation has captured the attention of the public and medical community as the next great challenge in assisted reproductive technology. The primary goal for those working in the fertility preservation realm has been spawned by the growing need to learn more about the reproductive health of individuals whose fecundity is compromised by events such as childhood treatments for cancer, autoimmune disease and other "gonadotoxic" therapies. After reviewing the status of this field over two and a half days of vigorous exchange of primary data, current practices, and promising leads for future research, two inescapable conclusions were drawn: that in order to meet the challenges that lie ahead for fertility preservation, an intense effort in basic and clinical research will be required to fill voids in our current knowledge base; and that encouraging discovery for fertility preservation could lead to the next renaissance in ARTs in general. For this reason, we have dedicated a special issue of JARG to this emerging area.

We begin the issue with an authoritative summary of the meeting proceedings in order to provide our readership with the nuts and bolts of the field; this will introduce both the emerging language and the status of ARTs pertinent to FP. 
Next, to be fair and objective in our treatment of this subject, we've included several papers that represent the forefront of the field, spanning the role of clinical decisionmaking to latest insights into the biology of cryopreservation. It should be emphasized that JARG will continue to cover topics within the domain of FP in an attempt to critically assess patient outcomes relative to basic science findings. Finally, so that our readership can truly appreciate the breadth and depth of this field, we are honored to provide the meetings abstracts, which will provide a closer look into the field from the perspective of the many laboratories that are making significant contributions.
Fertility Preservation will play a crucial role in future developments in ARTs. If we look beyond the pressing and critical needs that young patients with cancer present to the team of physicians responsible for their reproductive health, a landscape is revealed that extends well beyond the purview of traditional ARTs. As it becomes increasingly clear that our bodies are subject to the forces of our environment, which include the chemical and physical insults we subject it to, so too will the need to view fertility preservation from a lifestyle perspective as we chronically manage our reproductive health, rather than an answer to the acute problems that afflict those with childhood disease. 